\title{
PERCEIVED STRESS IN PATIENTS WITH CORONARY ARTERY DISEASE DURING THE COVID-19 PANDEMIC
}

\author{
Márcia Schmidt ${ }^{1}$, Karine Schmidt ${ }^{1}$, Vitória Silva ${ }^{2}$, Filipa Waihrich ${ }^{1}$, \\ \& Alexandre Quadros ${ }^{1}$ \\ ${ }^{I}$ Graduate Program in Health Sciences: Cardiology, University Foundation of Cardiology (Brazil) \\ ${ }^{2}$ Institute of Cardiology of Rio Grande do Sul (Brazil)
}

\begin{abstract}
Introduction: The COVID-19 pandemic has brought many routine changes to the entire Brazilian population. On mental health, individuals were observed to be increasingly susceptible to developing symptoms such as psychological stress, anxiety, and depression due to social restrictions. The psychosocial factors exerted a fundamental role in the etiology and evolution of cardiovascular diseases (CVDs), with stress being one of these factors. Objective: To evaluate the self-perceived stress on arterial coronary disease patients during the COVID-19 pandemic. Methods: Coronary artery disease patients undergoing percutaneous coronary intervention in the period between February and May 2020 at a reference hospital in cardiology were considered eligible. The patients were interviewed about their self-perceived stress through phone calls from August to December 2020. The clinical characteristics were obtained through the electronic record. The stress was evaluated using a Perceived Stress Scale PSS-10. Those patients with a score higher than the PSS-10 average (16 points) were considered stressed. The patients were divided into groups with and without stress. The categorical variables were expressed through the frequency and percentage and analyzed by the chi-square test, and the continuous variables were described by average and standard deviation, then compared using a Student's t test. Results: A total of 501 patients were assessed for eligibility, and 200 were included. Forty-nine percent of the patients presented stress symptoms. The stressed patients were frequently younger and had a family history of premature coronary artery disease. The women were more often stressed than the men, even though both groups had a higher prevalence of stressed patients than non-stressed patients. Concerning the PSS-10 questions, the patients with stress were more frequently upset with something unexpected; they felt more often unable to control important matters in their lives, more nervous, and irritated than those without stress. They also referred to the greater difficulty they faced in controlling irritations in their lives. Finally, $50 \%$ of the stressed patients also felt very frequently that their problems had accumulated in such a manner that they could not solve them anymore, in comparison to $0.98 \%$ in the without-stress group. Conclusion: The patients with arterial coronary disease and self-perceived stress were younger and presented a family history of premature coronary disease. They had less control over their irritations and important issues in their lives, feeling overloaded and incapable of solving their problems compared to those without stress.
\end{abstract}

Keywords: Stress, psychological, pandemics, coronary artery disease, percutaneous coronary intervention.

\section{Introduction}

The COVID-19 pandemic has changed the reality of people's lives and will have long-lasting ramifications for the health of the population and the health system (Khera et al., 2020). The worsening of mental health has been documented worldwide in this period, with symptoms of post-traumatic stress, panic disorder, depression, anxiety, and self-perceived stress on the rise, even after adjustments for previous psychiatric illnesses and a history of childhood trauma, suggesting that the COVID-19 pandemic is having an independent effect on the mental health of the population (Mimoun et al., 2020; Rossi et al., 2020).

In patients with coronary artery disease (CAD), who belong to a risk group for COVID-19, stress is a potential known risk factor for cardiovascular events and mortality (Richardson et al., 2012). The more detailed assessment of stress in the midst of the COVID-19 pandemic may contribute to more targeted mental health care for these patients.

\section{Objective}

To evaluate the self-perceived stress in CAD patients during the COVID-19 pandemic. 


\section{Methods}

Cross-sectional study. CAD patients who have undergone percutaneous coronary intervention at a reference hospital in cardiology were considered eligible.

\subsection{Inclusion and exclusion criteria}

Inclusion criteria: Patients with CAD obstruction of one or more epicardial arteries, with at least $50 \%$ stenosis and/or left main disease with at least $50 \%$ of stenosis measured by catheterization (CATE) and who had undergone PCI (Cesar et al., 2014); Exclusion criteria: Patients who are more than 75 years old or patients with cognitive disability. We excluded patients who lived farther from the hospital ( $>48$ miles) because at the time this project was conducted, we intended to call them to an outpatient clinic.

\subsection{Logistics}

Patients with CAD who underwent percutaneous coronary intervention in the period between February and May 2020 were sequentially assessed for eligibility according to the list of PCIs performed by the cath lab of a cardiology reference center in southern Brazil. Patients were interviewed about their own perceived stress through telephone calls from August to December 2020 by a trained investigator. All participants provided free and informed consent verbally by telephone to the researcher and via SMS phone message.

\subsection{Assessment of self-perceived stress}

The stress was evaluated using a Perceived Stress Scale - PSS-10. PSS items were designed to assess the degree to which respondents found their lives unpredictable, uncontrollable, and overburdened. It consisted of 10 items that measured perceived stressful experiences over the previous month with a 5 -point Likert scale ranging from never $(=0)$, almost never $(=1)$, sometimes $(=2)$, fairly often $(=3)$, and very often (=4). It is a scale composed of six negative items $(1,2,3,6,9,10)$ and four positive items $(4,5,7,8)$. Possible PSS-10 scores range from 0 to 40 . A higher score indicates greater stress (Cohen et al., 1983; Siqueira Reis et al., 2010). Those patients with scores higher than the average for the Brazilian population on PSS-10 (16 points) were considered stressed (Siqueira Reis et al., 2010).

\subsection{Statistical analysis}

The patients were divided into groups with and without stress. The categorical variables were expressed as frequencies and percentages and analyzed by the chi-square test, and the continuous variables were described by average and standard deviation compared using a Student's t test. A two-tailed $\mathrm{P}$ value $\leq 0.05$ was considered to indicate statistical significance. All analyses were performed using IBM $®$ SPSS $®$ software version 24.

\subsection{Ethical and legal considerations} committee.

This protocol is in accordance with the Helsinki declaration and was approved by the local ethics

\section{Results}

A total of 501 patients were assessed for eligibility, of whom 227 were excluded, mainly due to distance and age. Thus, we found 274 eligible patients. Of these, 56 were lost; 11 declined; and 7 died, leading to 200 participants. Of the general sample, $62.12 \%$ were male and $37.87 \%$ female. Forty-nine percent of the patients presented stress symptoms. Women have a $47.42 \%$ prevalence of stress, higher than men, at $28.71 \%$, although males were more prevalent in groups with and without stress $(p=0.008)$. The stressed patients were frequently younger $(\mathrm{p}=0.002)$ and with a premature family history of CAD $(\mathrm{p}=0.005)$ (Table1).

Concerning PSS-10 questions, patients with stress were more often upset by something unexpected $(60.20 \%$ vs. $8.82 \%)$; they more often felt unable to control important things in their lives $(37.75 \%$ vs. $0.98 \%)$ and were more nervous than those without stress $(63.26 \%$ vs. $9.80 \%)$. They felt less sure about their ability to handle their problems (40.81\% vs. $81.37 \%)$. They less often felt that things were going their way $(20.40 \%$ vs 47.05$)$, and they more often felt that they could not cope with everything they had to do $(33.7 \%$ vs. $1.96 \%)$. Only $22.44 \%$ of the stressed patients felt able to control their irritations, compared to $59.40 \%$ of those without stress. They felt less frequently that they were on top of things (16.32 vs. $55.88 \%$ ). Of the stressed people, $48.97 \%$ were angry about things that were outside of their control, which occurred only among $4.90 \%$ of those without stress. Finally, $50 \%$ of stressed people felt very often that problems had accumulated so much that they could not solve them, compared to $0.98 \%$ of those without stress. Figure 1 shows some items of the Perceived Stress Scale according to the groups. 
p-ISSN: 2184-2205 e-ISSN: 2184-3414 ISBN: 978-989-54815-5-2 @ 2021

Table 1. Characteristics of patients with coronary artery disease according to groups with and without stress.

\begin{tabular}{|c|c|c|c|}
\hline Characteristics & $\begin{array}{l}\text { With stress } \\
n=98(49 \%)\end{array}$ & $\begin{array}{c}\text { No stress } \\
n=102(51 \%)\end{array}$ & $p$ \\
\hline Age years & $58.74 \pm 9.83$ & $62.95 \pm 9.37$ & 0.002 \\
\hline \multicolumn{4}{|l|}{ Sex } \\
\hline $\begin{array}{l}\text { Female } n(\%) \\
\text { Male } n(\%)\end{array}$ & $\begin{array}{l}46(47.42) \\
51(52.57)\end{array}$ & $\begin{array}{l}29(28.71) \\
72(71.28)\end{array}$ & 0.008 \\
\hline Public Healthcare $n(\%)$ & $78 \quad(80.41)$ & $74(73.26)$ & 0.23 \\
\hline \multicolumn{4}{|l|}{ Risk Factors } \\
\hline Smoking $n(\%)$ & $24(24.48)$ & $18(17.64)$ & 0.24 \\
\hline Hypertension $n(\%)$ & $64(65.30)$ & $65(63.72)$ & 0.82 \\
\hline Diabetes Mellitus n (\%) & $35(35.71)$ & $35(34.31)$ & 0.84 \\
\hline Dyslipidemia $n(\%)$ & $46(46.93)$ & $38(37.25)$ & 0.17 \\
\hline Premature family history of $\mathrm{CAD} n(\%)$ & $30(30.92)$ & $19(18.62)$ & 0.05 \\
\hline Obesity $n(\%)$ & $29(29.59)$ & $24(23.76)$ & 0.35 \\
\hline \multicolumn{4}{|l|}{ Clinical History } \\
\hline Previous MI $n(\%)$ & $36(37.11)$ & $28(27.72)$ & 0.16 \\
\hline Previous CABG $n(\%)$ & $5(5.15)$ & $5(5.05)$ & 0.97 \\
\hline Previous PCI $n(\%)$ & $30(30.92)$ & $23(23.00)$ & 0.21 \\
\hline Current angina $n(\%)$ & $46(47.42)$ & $43(42.57)$ & 0.49 \\
\hline $\mathrm{HF} n(\%)$ & $12(12.37)$ & $8(7.92)$ & 0.30 \\
\hline Stroke $n(\%)$ & $4(4.12)$ & $2(1.98)$ & 0.38 \\
\hline Chronic kidney failure $n(\%)$ & $4(4.12)$ & 0 & 0.06 \\
\hline \multicolumn{4}{|l|}{ Angiographic aspects } \\
\hline \multicolumn{4}{|l|}{ Number of injured vessels } \\
\hline 1. $n(\%)$ & $56(57.14)$ & $60(58.82)$ & \multirow{3}{*}{0.21} \\
\hline 2. $n(\%)$ & $27(27.55)$ & $19(18.62)$ & \\
\hline 3. $n(\%)$ & $15(15.30)$ & $23(22.54)$ & \\
\hline
\end{tabular}

CAD-Coronary artery disease; MI-myocardial infarction; CABG-Coronary artery bypass graft; PCI-percutaneous coronary intervention; HF-heat failure;

Figure 1. Perceived Stress Scale items (PSS-10) according to groups with and without stress.
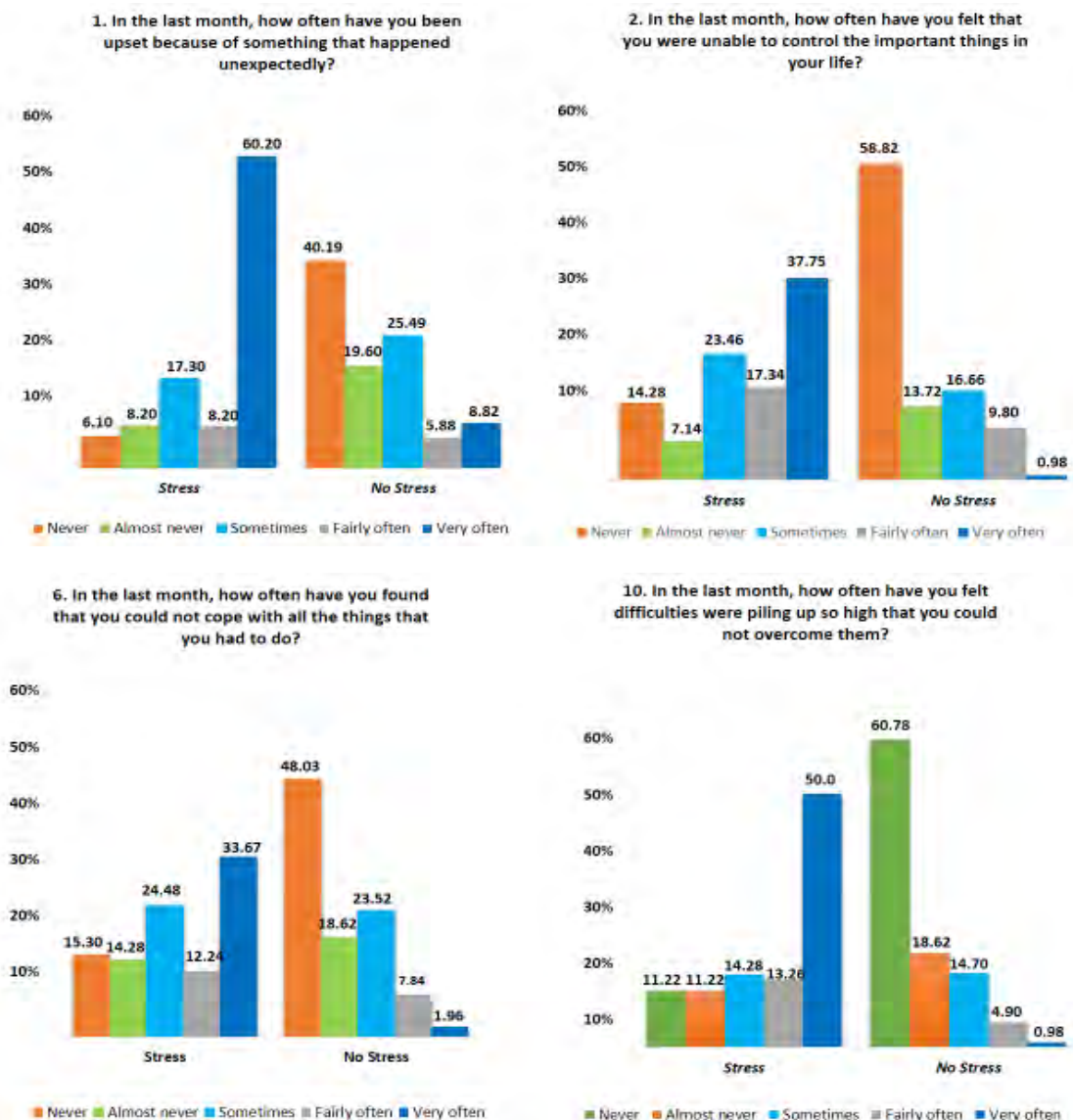

10. In the last month, how often have you felt
difficulties were piling up so high that you could not overcome them?

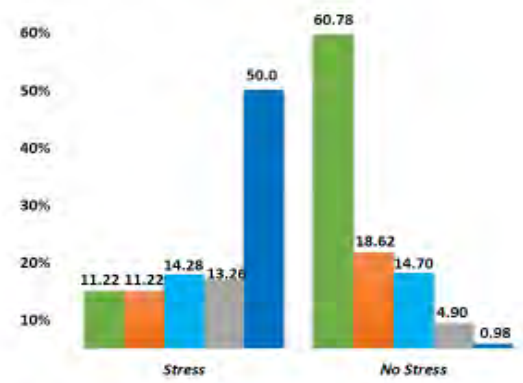

= Never - Almost never $=$ Sometimes $\equiv$ Fairly often $=$ Very often 


\section{Discussion}

In our study, we found a stress prevalence of $49 \%$ among patients, compared to $29.6 \%$ described in the general population in this pandemic period (Salari et al., 2020). It is possible that the knowledge among cardiovascular patients that they belong to the group at highest risk of complications for COVID-19 causes a constant state of alertness and an intensified concern in the adoption of care measures to prevent COVID-19 contamination. This reality can be considered an additional stressor at this time of pandemic in relation to the general population. These data deserve attention, since stress is known to be a risk factor for greater cardiovascular morbidity and mortality in the population with CAD (Richardson et al., 2012).

We observed in this study that the stressed patients were younger, and women had a higher prevalence of stress than men, which is in line with what has been documented in the current and the pre-pandemic scenario (Rossi et al., 2020; Xu et al., 2015). Considering that the patients with stress were younger and more likely to be of working age than those without stress, we can consider the hypothesis that they may have suffered more directly the economic impacts of the pandemic, with temporary or permanent loss of employment. The interruption of professional activity in the pandemic has been reported as a factor that can generate loss of confidence, self-esteem, and control, especially when the person perceives himself as a burden on society and feels a lack of belonging that contributes to emotional suffering (Brooks et al., 2020).

In our study, although stressed patients showed confidence in their ability to deal with their personal problems, most of them felt unable to control important things in life, indicating a feeling of helplessness, which is reinforced by their concern that they could not deal with everything they had to do. Stressed patients also seemed to suffer from anguish for the things they could not control and returned that sentiment in the form of hostility. This revolt is evidenced by more frequent annoyance in unexpected situations and anger at things that were out of control. The correlation between higher levels of self-perceived stress and greater hostility has been demonstrated in this period and seems to be mediated by social support, which reinforces the need for social bonds to achieve better emotional management in crisis situations and when facing high levels of stress such as the pandemic (Duan et al., 2020). In addition, we can assume that people become irritated when reality falls short of expectations, which is demonstrated by the frustration of most people who become stressed by things never happening in the expected way. This generates greater nervousness and less ability to control life's irritations. One study demonstrated a strong inverse correlation between perceived stress levels and sense of control, which confirms our findings that participants felt greater stress and less emotional and behavioral control (Mimoun et al., 2020). In addition, the feeling among half of the stressed people that they were accumulating problems to the point of not being able to resolve them indicates a stage of emotional exhaustion, a situation of greater vulnerability for less control in the face of stressful events, especially in individuals with negative emotional coping skills (Yan et al., 2020).

The pandemic caused several stressful events such as fear of infection with the new coronavirus; hospitalization; the death of loved ones by COVID-19; relationship difficulties; changes in work routines; and financial, supply, or housing problems (Lai et al., 2020; Mimoun et al., 2020; Rossi et al., 2020). In addition, long-term social isolation, frustration, boredom and lack of adequate information on the part of public health authorities have also been documented as stressful events (Brooks et al., 2020; Lai et al., 2020). All of these situations can increase stress even more in patients suffering from heart disease. This group of patients was also potentially affected by another stressor, adopted to reduce the spread of Sars-Cov-2 and redirect resources to care for patients with COVID-19: up to $80 \%$ of elective interventions were postponed indefinitely, first in Italy and then in most countries, with only emergency cardiac procedures being performed. In addition, the fear of contagion from the coronavirus has been reported as one of the factors in decreased attendance due to cardiovascular causes in emergencies, with studies from Europe and the United States reporting a more than 50\% reduction in hospital admissions due to acute coronary syndrome (Garcia et al., 2020; Stefanini et al., 2020).

One of the limitations of this study is that, to date, we do not have a control group, either of cardiovascular patients outside the pandemic period or of a general sample of the local population during the pandemic. Our future perspective is to evaluate, during the pandemic, participants without coronary heart disease for comparison with the results presented in this article. We intend to survey a group of coronary patients outside the pandemic period in the future. However, we emphasize that our objective was to evaluate and report these results as soon as possible, aiming at an early intervention in these patients, in view of the strong correlation between high stress and greater morbidity and mortality in patients with cardiovascular disease. This study allowed the prioritization of psychological care for women and those with a family history of coronary heart disease. 
In conclusion, the patients with arterial coronary disease and self-perceived stress are younger, female, and presented a family history of premature coronary disease. They have less control over their irritations and important issues in their lives, feeling overloaded and incapable of solving their problems compared to those without stress. A more detailed understanding of the characteristics of stress in patients with CAD during the COVID-19 pandemic period presented in this article may contribute to a more focused and assertive approach to the management of stress in this risk group.

\section{References}

Brooks, S. K., Webster, R. K., Smith, L. E., Woodland, L., Wessely, S., Greenberg, N., \& Rubin, G. J. (2020). The psychological impact of quarantine and how to reduce it: rapid review of the evidence. The Lancet.

Cesar, L., Ferreira, J., Armaganijan, D., Gowdak, L., Mansur, A., Bodanese, L., Sposito, A., Sousa, A., Chaves, A., \& Markman, B. (2014). Diretriz de doença coronária estável. Arquivos Brasileiros De Cardiologia.

Cohen, S., Kamarck, T., \& Mermelstein, R. (1983). A global measure of perceived stress. Journal of health and social behavior, 385-396.

Duan, H., Yan, L., Ding, X., Gan, Y., Kohn, N., \& Wu, J. (2020). Impact of the COVID-19 pandemic on mental health in the general Chinese population: Changes, predictors and psychosocial correlates. Psychiatry research, 293, 113396.

Garcia, S., Albaghdadi, M. S., Meraj, P. M., Schmidt, C., Garberich, R., Jaffer, F. A., Dixon, S., Rade, J. J., Tannenbaum, M., \& Chambers, J. (2020). Reduction in ST-segment elevation cardiac catheterization laboratory activations in the United States during COVID-19 pandemic. Journal of the American College of Cardiology, 75(22), 2871-2872.

Khera, A., Baum, S. J., Gluckman, T. J., Gulati, M., Martin, S. S., Michos, E. D., Navar, A. M., Taub, P. R., Toth, P. P., \& Virani, S. S. (2020). Continuity of care and outpatient management for patients with and at high risk for cardiovascular disease during the COVID-19 pandemic: A scientific statement from the American Society for Preventive Cardiology. American Journal of Preventive Cardiology, 1, 100009

Lai, J., Ma, S., Wang, Y., Cai, Z., Hu, J., Wei, N., Wu, J., Du, H., Chen, T., \& Li, R. (2020). Factors associated with mental health outcomes among health care workers exposed to coronavirus disease 2019. JAMA network open, 3(3), e203976-e203976.

Mimoun, E., Ben Ari, A., \& Margalit, D. (2020). Psychological aspects of employment instability during the COVID-19 pandemic. Psychological Trauma: Theory, Research, Practice, and Policy, 12(S1), S183.

Richardson, S., Shaffer, J. A., Falzon, L., Krupka, D., Davidson, K. W., \& Edmondson, D. (2012). Meta-analysis of perceived stress and its association with incident coronary heart disease. The American journal of cardiology, 110(12), 1711-1716.

Rossi, R., Socci, V., Talevi, D., Mensi, S., Niolu, C., Pacitti, F., Di Marco, A., Rossi, A., Siracusano, A., \& Di Lorenzo, G. (2020). COVID-19 pandemic and lockdown measures impact on mental health among the general population in Italy. Frontiers in Psychiatry, 11.

Salari, N., Hosseinian-Far, A., Jalali, R., Vaisi-Raygani, A., Rasoulpoor, S., Mohammadi, M., Rasoulpoor, S., \& Khaledi-Paveh, B. (2020). Prevalence of stress, anxiety, depression among the general population during the COVID-19 pandemic: a systematic review and meta-analysis. Globalization and health, 16(1), 1-11.

Siqueira Reis, R., Ferreira Hino, A. A., \& Romélio Rodriguez Añez, C. (2010). Perceived stress scale: reliability and validity study in Brazil. Journal of health psychology, 15(1), 107-114.

Stefanini, G. G., Azzolini, E., \& Condorelli, G. (2020). Critical organizational issues for cardiologists in the COVID-19 outbreak: a frontline experience from Milan, Italy. Circulation, 141(20), 1597-1599.

Xu, X., Bao, H., Strait, K., Spertus, J. A., Lichtman, J. H., D'onofrio, G., Spatz, E., Bucholz, E. M., Geda, M., \& Lorenze, N. P. (2015). Sex differences in perceived stress and early recovery in young and middle-aged patients with acute myocardial infarction. Circulation, CIRCULATIONAHA. 114.012826.

Yan, L., Gan, Y., Ding, X., Wu, J., \& Duan, H. (2020). The relationship between perceived stress and emotional distress during the COVID-19 outbreak: Effects of boredom proneness and coping style. Journal of anxiety disorders, 77, 102328. 